\title{
Impact of the occurrence of new lesions on the survival of patients who undergo chemotherapy for metastatic colorectal cancer
}

\author{
MASATSUNE SHIBUTANI, KIYOSHI MAEDA, HISASHI NAGAHARA, TATSUNARI FUKUOKA, \\ SHINJI MATSUTANI, SHINICHIRO KASHIWAGI, KOSEI HIRAKAWA and MASAICHI OHIRA
}

Department of Surgical Oncology, Osaka City University Graduate School of Medicine, Osaka, Osaka 545-8585, Japan

Received July 13, 2018; Accepted November 21, 2018

DOI: $10.3892 / \mathrm{mco} .2018 .1778$

\begin{abstract}
In clinical practice, the efficacy of chemotherapy for metastatic colorectal cancer (mCRC) is typically evaluated according to the Response Evaluation Criteria in Solid Tumours (RECIST) criteria, and an appropriate treatment plan is determined. In the case of progressive disease (PD), the components of the treatment are altered; however, PD, as defined by the RECIST criteria, includes various types of progression. While detailed consideration regarding the impact of the growth pattern of measurable target lesions on survival has been performed, the impact of the occurrence of new lesions on survival is unclear. The aim of the present study was to assess the impact of the occurrence of new lesions on the survival of patients who underwent chemotherapy for mCRC. Among the patients who received doublet chemotherapy for mCRC as a first-line treatment between 2008 and 2016, 81, who stopped the chemotherapy due to PD, were enrolled in the present study. The types of progression were classified according to the definitions of RECIST. Subsequently, the following criteria were considered: The growth of measurable target lesions, the occurrence of new lesions and the unequivocal progression of non-target disease. Furthermore, the developing patterns of new lesions were also assessed. The association between the type of progression and
\end{abstract}

Correspondence to: Dr Masatsune Shibutani, Department of Surgical Oncology, Osaka City University Graduate School of Medicine, 1-4-3 Asahi-machi, Abeno-ku, Osaka, Osaka 545-8585, Japan

E-mail: fbxbj429@ybb.ne.jp

Abbreviations: mCRC, metastatic colorectal cancer; RECIST, Response Evaluation Criteria in Solid Tumours; PD, progressive disease; CT, computed tomography; PS, performance status; CI, confidence interval; SD, stable disease; PR, partial response; RAS, rat sarcoma; FOLFOX, 5-fluorouracil + leucovorin + oxaliplatin; CapeOX, capecitabine + oxaliplatin; FOLFIRI, 5-fluorouracil + leucovorin + irinotecan; SOX, S-1+ oxaliplatin; IRIS, S-1+ irinotecan

Key words: colorectal cancer, chemotherapy, progressive disease, new lesion, prognosis the survival after the failure of the first-line chemotherapy was explored. Forty (49.4\%) patients only experienced growth of measurable target lesions, $41(50.6 \%)$ of the patients had new lesions and $3(3.7 \%)$ of the patients had unequivocal progression of non-target disease. The survival rate from the discontinuation of first-line chemotherapy in patients with new lesions was significantly worse than that in patients without new lesions $(\mathrm{P}=0.0068)$; however, the developing patterns of new lesions were not associated with survival. Regardless of the developing patterns of new lesions, patients who stopped chemotherapy due to new lesions had worse survival rates from the discontinuation of first-line chemotherapy compared with those who stopped chemotherapy due only to the growth of measurable target lesions. Because the occurrence of new lesions represents severe progressive disease, patients with new lesions may require more intensive chemotherapy. The type of progression may be useful information for selecting the appropriate treatment following the failure of first-line chemotherapy.

\section{Introduction}

As there can be major differences in the effect of chemotherapy even when using the same regimen, it is necessary to evaluate the therapeutic effect at regular intervals. Therefore, some indices are necessary to evaluate the therapeutic effect objectively. In clinical practice, we usually evaluate the efficacy of chemotherapy for metastatic colorectal cancer (mCRC) according to the Response Evaluation Criteria in Solid Tumors (RECIST) criteria and determine an appropriate treatment plan (e.g., whether the treatment currently being administered should be continued or changed to another treatment); however, the RECIST criteria were made in order to assess the objective therapeutic response of new drugs or new regimen, not to judge whether the treatment should be continued in individual cases $(1,2)$. Therefore, whether or not the RECIST criteria are a perfect evaluation tool is unclear, although these criteria are frequently used in routine practice.

In the case of progressive disease (PD), we change the components of the treatment; however, PD as defined by the RECIST criteria includes three types of progression as follows: i) Growth of measurable target lesion(s); ii) the occurrence of new lesions; and iii) unequivocal progression of non-target disease. While detailed consideration about the 
impact of the growth pattern of measurable target lesions, such as the growth speed, on the survival has been performed Specifically, Suzuki et al (3) reported that the change in tumor diameter in the first two weeks from the initiation of the first-line chemotherapy for mCRC had a strong ability to predict the survival. Litière et al (4) examined the significance of the tumor growth of target lesions as the worst \% change (\%/week) and worst rate of increase ( $\mathrm{mm} /$ week) from the nadir and revealed that the tumor growth rate correlated with the survival outcomes in various malignancies. By contrast, there has not been sufficient discussion about the significance of the occurrence of new lesions and the impact of the occurrence of new lesions on the survival is unclear. Furthermore, few reports have examined the impact of the development patterns of new lesions on the survival.

The aim of this study was to assess the impact of the occurrence of new lesions on the survival in patients who underwent chemotherapy for $\mathrm{mCRC}$ in order to refine the categorization of tumor progression.

\section{Materials and methods}

Patients. Among the patients who received doublet chemotherapy for unresectable $\mathrm{mCRC}$ as a first-line treatment at the Department of Surgical Oncology of Osaka City University between 2008 and 2016, 81 who stopped the first-line chemotherapy due to PD were enrolled in this study (Fig. 1). When grade $\geq 3$ adverse events occurred, chemotherapy was stopped. When restarting therapy in such cases, the dose was reduced by approximately $20 \%$. No patients had radiofrequency ablation (RFA) or transcatheter arterial embolization (TACE) after failure of the first-line treatment. All baseline evaluations were performed within four weeks before the initiation of first-line chemotherapy, and the tumor response was evaluated every eight weeks by computed tomography (CT). When the treatment was interrupted by adverse events, the timing of the evaluation was delayed. Such a delay was allowed provided it was within one month.

The evaluation of the objective tumor response. The objective tumor response was assessed according to the RECIST 1.1 criteria (2). In brief, at baseline, the lesions were categorized into measurable and non-measurable lesions. For tumor lesions, measurable lesions were defined as those that could be accurately measured in at least 1 dimension with a long axis of $>10 \mathrm{~mm}$ on CT (for lymph nodes, $>15 \mathrm{~mm}$ on CT). Non-measurable lesions were defined as the lesions other than the measurable ones, including small lesions and truly non-measurable lesions, such as ascites.

When evaluating the measurable lesions, all lesions up to a maximum of five total (maximum of two per organ) were identified as the target lesions, and the sum of the diameters for all of the target lesions was calculated. An increase of $>20 \%$ in the sum of the target lesions compared with the smallest sum on the study and an absolute increase of $\geq 5 \mathrm{~mm}$ was judged to be PD. For non-target lesions, unequivocal progression, such as a rapid and significant increase in ascites, was judged to be PD. The appearance of new lesions was also judged to be PD. In some cases, the criteria for PD overlapped.
The evaluation of the developing patterns of new lesions. We examined the developing patterns of new lesions and assessed the impact of these factors on the survival. The points of consideration were as follows: i) The number of new lesions; ii) the lesions present in new organs that had not been affected by metastasis; and iii) equivocal lesions (i.e., small lesions) judged as truly new disease according to a follow-up examination.

Statistical analyses. The significance of the correlations between the type of progression and the clinicopathological factors was analyzed using the Chi-square test. The survival time from the discontinuation of first-line chemotherapy was defined as the interval between the date of the failure of the first-line chemotherapy due to PD and the date of the death from any cause or the last follow-up. Survival curves were made using the Kaplan-Meier method. Differences in the survival curves were assessed using the log-rank test. A multivariate Cox proportional hazard model was applied to evaluate the prognostic factors associated with the survival. Factors with a P-value of $<0.1$ on the univariate analysis were included in the multivariate analysis. All of the statistical analyses were performed using the SPSS statistical software program, version 19.0 (IBM Corp., Armonk, NY, USA). P<0.05 was considered to indicate a statistically significant difference.

\section{Results}

Patient characteristics. The patient characteristics are summarized in Table I. The patient population consisted of 38 males and 43 females, with a median age of 63.0 years (range: 18 to 89 years). Twenty-six patients had primary tumors located in the right side, and 55 had primary tumors located in the left side. Fifty-four patients had single-organ metastasis, and 27 had multiple organs affected by metastases. All of the patients underwent combination chemotherapy with oxaliplatin, irinotecan plus 5-fluorouracil/leucovorin, or the prodrug of 5-fluorouracil as first-line chemotherapy. Forty patients received FOLFOX, 23 received CapeOX, 15 received FOLFIRI, and 3 received SOX. Fifty-six patients $(69.1 \%)$ underwent chemotherapy combined with molecular-targeted therapy. The objective response rate of first-lime chemotherapy was $24.7 \%$, and the disease control rate of first-line chemotherapy was $53.0 \%$.

The criteria for PD were as follows: $41(50.6 \%)$ patients had new lesions, 40 (49.4\%) only experienced growth of measurable target lesions, and $3(3.7 \%)$ had unequivocal progression of non-target disease. Among the 41 patients with the occurrence of new lesions, there were only 4 patients $(9.8 \%)$ who did not experience the growth of measurable target lesions. All patients with unequivocal progression of non-target disease also had new lesions. According to the definition of the Eastern Cooperative Oncology Group performance status (PS) at the failure of firstline chemotherapy, 51 patients were classified as having a PS of 0,21 as having a PS of 1, 3 as having a PS of 2 and 6 as having a PS of 3. Among these 81 patients, 17 (21.0\%) could not transfer to second-line chemotherapy after the discontinuation of first-line chemotherapy. Details about the developing patterns of new lesions were as follows (Table II): 19 (46.3\%) patients had new lesions present at new organs that had not been affected 
Table I. Patients' characteristics.

Variable Number

Age (years)

Median (range)

$63.0(18-89)$

Sex

Male

Female

Location of primary tumor

Right side

Left side

Histological type

Well-/moderately-differentiated

Poorly differentiated, Mucinous

RAS status

Wild-type

Mutant type

Unknown

Resection of the primary tumor

Positive

Negative

Local recurrence of rectal cancer

Positive

Negative

Number of organs affected by metastasis at the initiation of first-line chemotherapy

One organ

Multiple organs

Peritoneal dissemination at

the initiation of first-line chemotherapy

Negative

Positive

First-line chemotherapy regimen

FOLFOX

CapeOX

FOLFIRI

SOX

Molecular targeted therapy at

first-line chemotherapy

Bevacizumab

Cetuximab

Panitumumab

None

Tumor response to first-line chemotherapy

PR

$\mathrm{SD}$

PD

Criteria for progressive disease

Occurrence of new lesions

Only growth of measurable lesions

Unequivocal progression of non-target disease
Table I. Continued.

\begin{tabular}{|c|c|}
\hline Variable & Num \\
\hline \multicolumn{2}{|l|}{$\begin{array}{l}\text { Performance status at } \\
\text { the failure of first-line chemotherapy }\end{array}$} \\
\hline 0 & 51 \\
\hline 1 & 21 \\
\hline 2 & 3 \\
\hline 3 & 6 \\
\hline \multicolumn{2}{|l|}{ Second-line chemotherapy regimen } \\
\hline FOLFIRI & 41 \\
\hline FOLFOX & 8 \\
\hline IRIS & 5 \\
\hline CPT-11 & 2 \\
\hline CapeOX & 2 \\
\hline Capecitabine & 2 \\
\hline SOX & 1 \\
\hline S-1 & 1 \\
\hline Only molecular-targeted therapy & 2 \\
\hline None (best supportive care) & 17 \\
\hline \multicolumn{2}{|l|}{$\begin{array}{l}\text { Molecular-targeted therapy } \\
\text { at second-line chemotherapy }\end{array}$} \\
\hline Bevacizumab & 36 \\
\hline Cetuximab & 6 \\
\hline Panitumumab & 3 \\
\hline None & 19 \\
\hline
\end{tabular}

RAS, rat sarcoma; FOLFOX, 5-fluorouracil + leucovorin + oxaliplatin; CapeOX, capecitabine + oxaliplatin; FOLFIRI, 5fluorouracil + leucovorin + irinotecan; SOX, S-1+ oxaliplatin; IRIS, S-1+ irinotecan; PR, partial response; SD, stable disease; PD, progressive disease.

Table II. Details of the developing pattern of new lesions.

Variable Number (\%)

Lesions present at new organs that had not been affected by metastasis

Negative

Positive

Number of new lesions

$\geq 3$

Equivocal lesions judged to be truly new disease by a follow-up examination

Negative

Positive by metastasis, and $22(53.7 \%)$ had new lesions present only at the organs that had already been affected by metastasis. Six (14.6\%) patients had only 1 new lesion, 8 (19.5\%) had 2 new lesions, and 
Table III. Correlations between the type of progression and the clinicopathological factors.

\begin{tabular}{lccc}
\hline Variable & $\begin{array}{c}\text { Only growth of } \\
\text { measurable target lesions }\end{array}$ & $\begin{array}{c}\text { New } \\
\text { lesions }\end{array}$ & $\begin{array}{c}\text { Unequivocal progression } \\
\text { of non-target disease }\end{array}$ \\
\hline
\end{tabular}

Age (years)

$<63$

23

$\geq 63$

17

17

Male

Female

23

27

Right side

Left side

13

36

4

Well-/moderately-differentiated

Poorly differentiated, mucinous

RAS status

Wild-type

Mutant type

18

16

27

13

2

Peritoneal dissemination at the

initiation of first-line chemotherapy

Tumor response to first-line

chemotherapy

Second-line chemotherapy

17

21

19

19

27

11

34

4

11

19

1

2

0.440

0.629

0.402
30

10

27

13

15

23

Negative

Positive

$\mathrm{PR} / \mathrm{SD}$

PD

29

11

$\geq 1$

Performance status at the failure

of first-line chemotherapy

0

Present

35

5

Absent (best supportive care)

(1)

31

7

1

2

0.156

PR, partial response; SD, stable disease; PD, progressive disease; RAS, rat sarcoma.
$27(65.9 \%)$ had 3 or more new lesions. Four (9.8\%) patients have equivocal lesions that were judged to be truly new disease by a follow-up examination.

The median follow-up period after the discontinuation of firstline chemotherapy was 9.3 months (range: 0.2 to 77.4 months). Sixty-nine patients $(85.2 \%)$ died during the follow-up period.

Correlations between the type of progression and clinicopathological factors. The correlations between the type of progression and clinicopathological factors are shown in Table III. The disease control rate of first-line chemotherapy in the patients with new lesions was significantly higher than that in the patients without new lesions $(\mathrm{P}=0.036)$. Although there were no relationships between the type of progression and PS at the failure of first-line chemotherapy, the transfer ratio of second-line chemotherapy after the failure of first-line chemotherapy in the patients with new lesions tended to be lower than that in the patients without new lesions $(\mathrm{P}=0.078)$. There were no significant differences in the occurrence of new lesions by relative dose intensity.

Survival analyses according to the type of progression. The survival rate from the discontinuation of first-line 
Patients who underwent chemotherapy for unresectable $\mathrm{mCRC}$ as a first-line treatment $(\mathrm{n}=223)$

Excluded $(\mathrm{n}=49)$

Patients who underwent 5-FU monotherapy

Patients who underwent doublet chemotherapy $(n=174)$

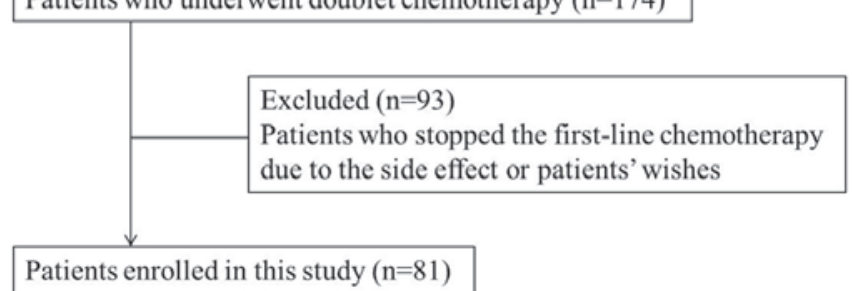

Figure 1. Patient selection flow diagram. 5-FU, 5-fluorouracil; mCRC, metastatic colorectal cancer.

chemotherapy in patients with new lesions was significantly worse than that in patients without new lesions $(\mathrm{P}=0.0068$; Fig. 2A). Furthermore, even in the analysis limited to the patients without unequivocal progression of non-target disease, the survival rate from the discontinuation of first-line chemotherapy in patients with new lesions was significantly worse than that in patients without new lesions $(\mathrm{P}=0.0038$; Fig. 2B). In addition, in the analysis targeting the 38 patients with new lesions but without unequivocal progression of non-target disease, the existence of the growth of measurable target lesions was found not to be associated with the survival (Fig. 2C).

Prognostic factors influencing the overall survival after treatment failure of first-line chemotherapy. The correlations between the survival time from the discontinuation of first-line chemotherapy and the various clinicopathological factors are shown in Table IV. According to the results of a univariate analysis, the survival rate from the discontinuation of first-line chemotherapy was significantly associated with the histological type $(\mathrm{P}<0.001)$, the tumor response to first-line chemotherapy $(\mathrm{P}=0.025)$, the occurrence of new lesions $(\mathrm{P}=0.008), \mathrm{PS}$ at the failure of first-line chemotherapy $(\mathrm{P}<0.001)$ and inability to transfer to second-line chemotherapy $(\mathrm{P}=0.001)$ and tended to be associated with the location of the primary tumor $(\mathrm{P}=0.095)$. A multivariate analysis indicated that the histological type (hazard ratio: $4.667,95 \%$ confidence interval: $1.810-12.032, \mathrm{P}=0.001$ ), the occurrence of new lesions (hazard ratio: 1.946, 95\% confidence interval: 1.146-3.304, $\mathrm{P}=0.014$ ), $\mathrm{PS}$ at the failure of first-line chemotherapy (hazard ratio: $2.289,95 \%$ confidence interval: $1.277-4.105, \mathrm{P}=0.005$ ) and inability to transfer to second-line chemotherapy (hazard ratio: $1.955,95 \%$ confidence interval: $1.020-3.746, \mathrm{P}=0.043$ ) were independent prognostic factors for the survival after the discontinuation of first-line chemotherapy.

Survival analyses according to developing patterns of new lesions. None of the developing patterns, such as the number of new lesions, the lesions present in new organs that had not

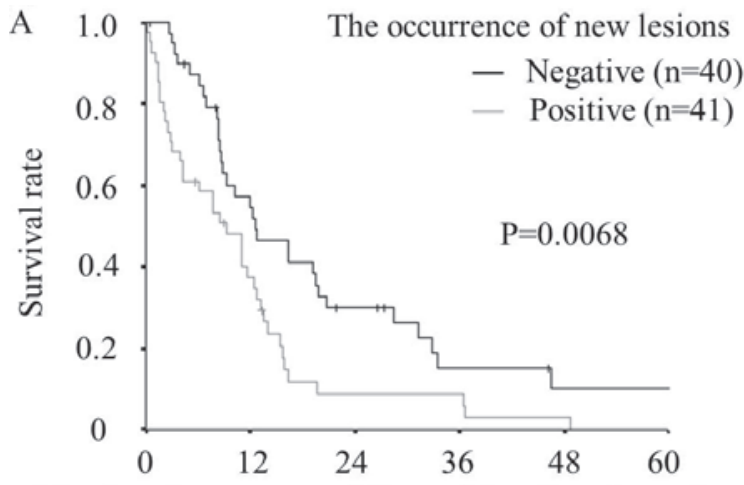

Months after treatment failure of first-line chemotherapy

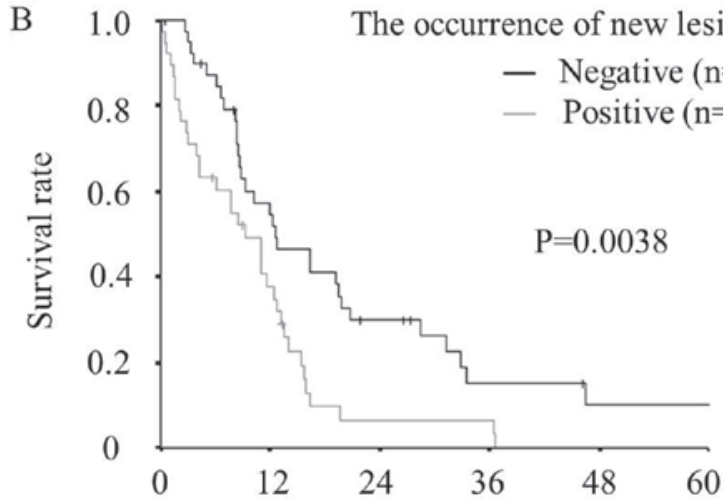

Months after treatment failure of first-line chemotherapy

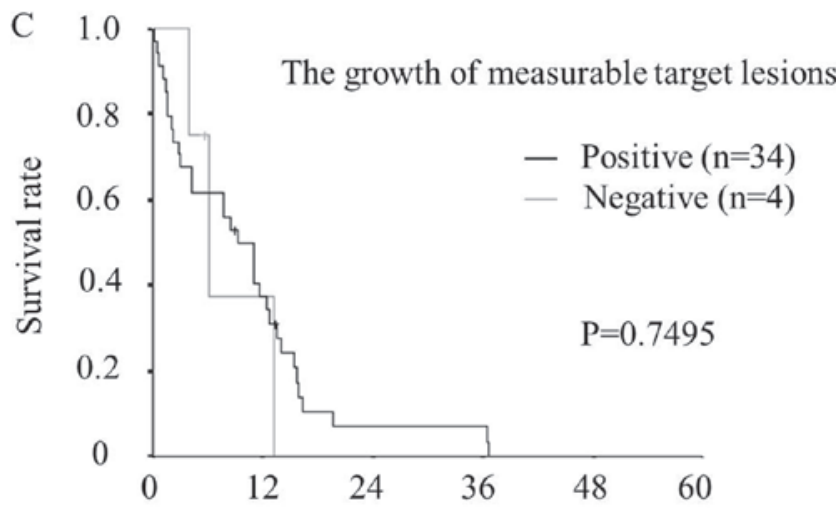

Months after treatment failure of first-line chemotherapy

Figure 2. Kaplan-Meier survival curves for the overall survival according to the occurrence of the new lesions. (A) In the analysis of all cases, the overall survival rate in patients with new lesions was significantly worse than that in patients without new lesions ( $\mathrm{P}=0.0068)$. (B) In the analysis limited to the patients without unequivocal progression of non-target disease, the overall survival rate in patients with new lesions was significantly worse than that in patients without new lesions $(\mathrm{P}=0.0038)$. (C) In the analysis targeting the 38 patients with new lesions but without any unequivocal progression of non-target disease, the existence of the growth of measurable target lesions was not associated with survival $(\mathrm{P}=0.7495)$.

been affected by metastasis, or equivocal lesions judged as truly new disease by a follow-up examination, was found to be associated with the survival (Fig. 3).

\section{Discussion}

In this study, the types of progression were found to affect the survival after treatment failure of first-line chemotherapy. The 
Table IV. Correlations between the survival time from the discontinuation of first-line chemotherapy and various clinicopathological factors.

\begin{tabular}{|c|c|c|c|c|c|c|}
\hline \multirow[b]{2}{*}{ Variable } & \multicolumn{3}{|c|}{ Univariate analysis } & \multicolumn{3}{|c|}{ Multivariate analysis } \\
\hline & Hazard ratio & $95 \% \mathrm{CI}$ & P-value & Hazard ratio & $95 \% \mathrm{CI}$ & P-value \\
\hline Age (years; $\geq 63$ vs. <63) & 1.347 & $0.836-2.169$ & 0.221 & & & \\
\hline Sex (female vs. male) & 1.207 & $0.750-1.943$ & 0.439 & & & \\
\hline Performance status ( $\geq 1$ vs. 0 ) & 1.632 & $0.825-3.226$ & 0.159 & & & \\
\hline Location of primary tumor (right side vs. left side) & 1.537 & $0.927-2.546$ & 0.095 & 1.724 & $0.952-3.123$ & 0.072 \\
\hline $\begin{array}{l}\text { Histological type (poorly differentiated, mucinous vs. } \\
\text { well-/moderately-differentiated) }\end{array}$ & 6.854 & 2.913-16.130 & $<0.001$ & 4.667 & $1.810-12.032$ & 0.001 \\
\hline RAS status (wild-type vs. mutant type) & 1.289 & $0.752-2.208$ & 0.355 & & & \\
\hline Local recurrence of rectal cancer (positive vs. negative) & 0.536 & $0.165-1.747$ & 0.301 & & & \\
\hline $\begin{array}{l}\text { Number of organs affected by metastasis ( } \geq 2 \text { vs. } 1 \text { ) at the } \\
\text { initiation of first-line chemotherapy }\end{array}$ & he 1.022 & $0.619-1.689$ & 0.932 & & & \\
\hline $\begin{array}{l}\text { Peritoneal dissemination (positive vs. negative) } \\
\text { at the initiation of first-line chemotherapy }\end{array}$ & 1.232 & $0.703-2.160$ & 0.467 & & & \\
\hline $\begin{array}{l}\text { Molecular targeted therapy at first-line chemotherapy } \\
\text { (without vs. with) }\end{array}$ & 1.147 & $0.701-1.875$ & 0.586 & & & \\
\hline $\begin{array}{l}\text { Tumor response to first-line chemotherapy } \\
\text { (PD vs. PR/SD) }\end{array}$ & 1.750 & $1.071-2.860$ & 0.025 & 1.135 & $0.661-1.949$ & 0.646 \\
\hline New lesions (positive vs. negative) & 1.928 & $1.189-3.125$ & 0.008 & 1.946 & $1.146-3.304$ & 0.014 \\
\hline $\begin{array}{l}\text { Performance status ( } \geq 1 \text { vs. } 0 \text { ) at the failure } \\
\text { of first-line chemotherapy }\end{array}$ & 2.454 & $1.500-4.017$ & $<0.001$ & 2.289 & $1.277-4.105$ & 0.005 \\
\hline Second-line chemotherapy (absent vs. present) & 2.568 & $1.456-4.530$ & 0.001 & 1.955 & $1.020-3.746$ & 0.043 \\
\hline
\end{tabular}

CI, confidence interval; PD, progressive disease; PR, partial response; SD, stable disease; RAS, rat sarcoma.

survival rate after treatment failure of first-line chemotherapy in patients who stopped chemotherapy due to the occurrence of new lesions was significantly worse than that in patients who stopped chemotherapy due only to the growth of measurable target lesions. The patients judged as having PD due to the occurrence of new lesions included those with unequivocal progression of non-target disease, defined as significant disease progression to stop treatment. Therefore, we reassessed the impact of the occurrence of new lesions on the survival after excluding the patients with unequivocal progression of non-target disease. Nevertheless, the survival rate in patients with the occurrence of new lesions was still significantly worse than that in patients with only the growth of measurable lesions.

We additionally assessed the impact of the developing patterns of new lesions on the survival after treatment failure of first-line chemotherapy. There was no significant difference in the prognosis between the patients with new lesions at new organs that had not been affected by metastasis and those with new lesions only at the organs that had already been affected by metastasis. Furthermore, the number of new lesions was revealed to have no influence on the survival. Moreover, even if the new lesions were deemed equivocal lesions at the time of occurrence that was judged to be truly new disease according to a follow-up examination, the impact of these lesions on the prognosis was equivalent to that of unequivocal lesions at the time of occurrence. All new equivocal lesions at the time of occurrence became unequivocal lesions within a short period of time in this study. There was no difference in the prognosis due to the size of the lesion at the time of occurrence. Whether a lesion was equivocal or unequivocal, it still represented the occurrence of a new lesion. Regardless of the number of new lesions, the organ in which new lesions occurred, or the size at the time of occurrence, the occurrence of a new lesion per se indicates that the current treatment cannot control the progression of disease.

The biological mechanism underlying the association of new lesions with a poor prognosis is unknown. However, speculations have been made. The disease control rate of first-line chemotherapy in the patients with new lesions was lower than that in those without new lesions. Furthermore, the transfer ratio of second-line chemotherapy in the patients with new lesions was also lower than in those without new lesions, as the condition of the patients with new lesions was likely to worsen shortly after the failure of first-line chemotherapy. For these reasons, the occurrence of new lesions likely indicates intensive disease progression. Such disease progression results in the inability to control micrometastases, which subsequently leads to the occurrence of new lesions.

Our data suggest that the occurrence of new lesions represented severe progressive disease, regardless of the developing pattern. Therefore, we should treat patients with new lesions as having a high possibility of a poor prognosis. Patients with new lesions may need intensive chemotherapy, such as doublet chemotherapy in a full dose and anti-EGFR antibody (when 


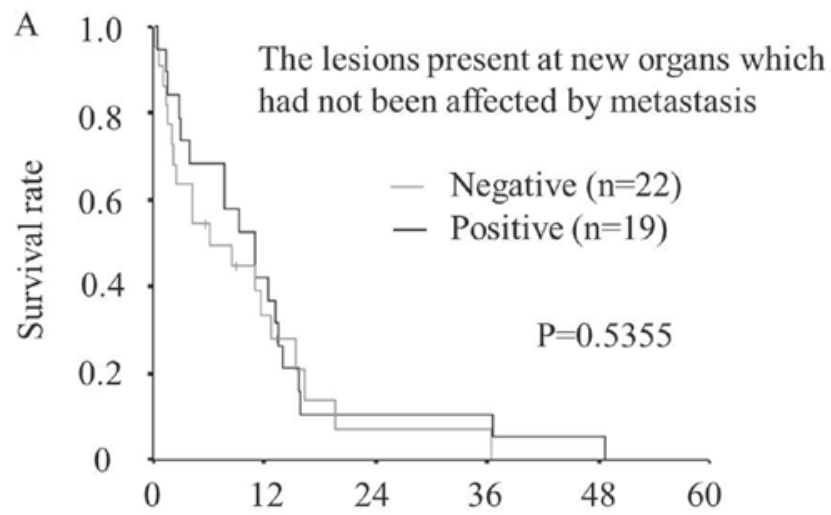

Months after treatment failure of first-line chemotherapy

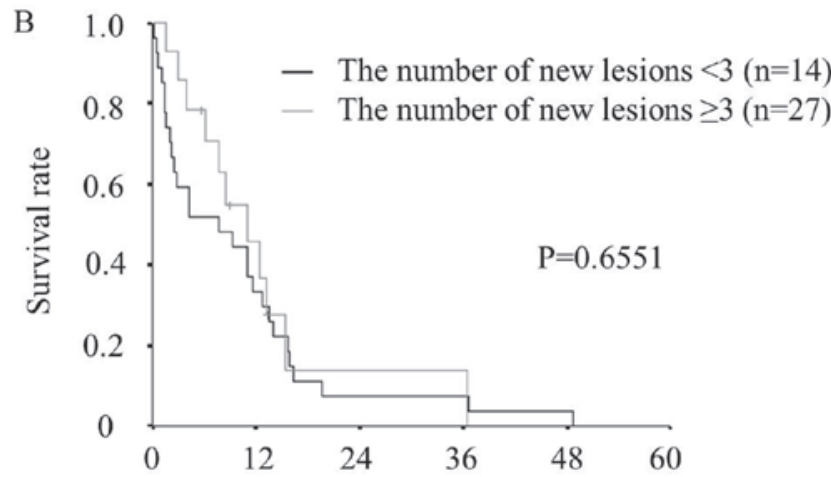

Months after treatment failure of first-line chemotherapy

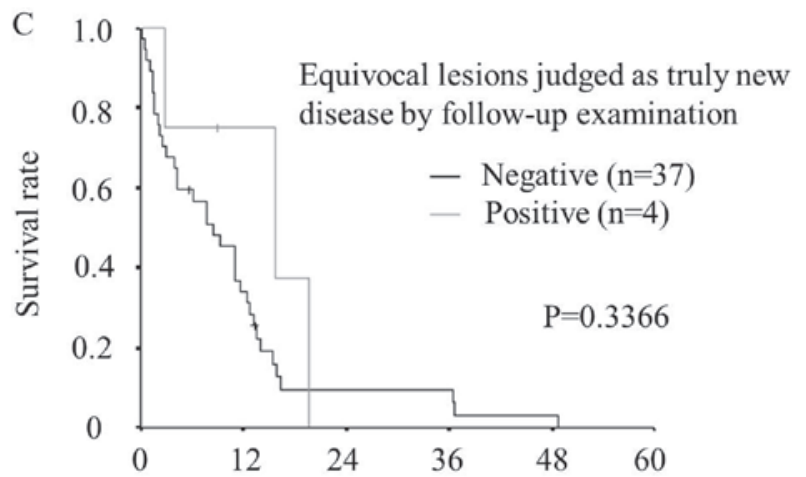

Months after treatment failure of first-line chemotherapy

Figure 3. Kaplan-Meier survival curves for the overall survival according to the developing patterns of new lesions. (A) There was no significant difference in the prognosis between patients with new lesions present at new organs that had not been affected by metastasis and patients with new lesions present only in the organs that had already been affected by metastases $(\mathrm{P}=0.5355)$. (B) There was no significant difference in the prognosis between patients with one or two new lesions and patients with three or more new lesions $(\mathrm{P}=0.6551)$. (C) There was no significant difference in the prognosis between patients with only equivocal lesions judged to be truly new disease by a follow-up examination and patients with obvious new lesions at the time of occurrence $(\mathrm{P}=0.3366)$.

RAS wild-type and anti-EGFR antibody were not used), with the goal of cytoreduction rather than disease control; however, it is also important to bear in mind that the condition of patients with new lesions may worsen quickly, resulting in the need to shift to best supportive care.

The overall survival has been used as the primary endpoint in many phase III clinical trials $(5,6)$, as it has been considered to be the most convincing measure of drug efficacy and clinical benefit (7); however, effectively evaluting the overall survival requires a large number of cases and a long follow-up period. To resolve these issues, the progression-free survival has also been used as a surrogate primary endpoint (8-10), based on reports that the progression-free survival had a strong correlation with the overall survival $(11,12)$; however, the progression-free survival has occasionally been found to be unreliable as a surrogate endpoint for the overall survival (13-15). Gill et al (7) suggested that the effects of subsequent-line therapies might underlie such conflicting findings. In addition, the results obtained in this study suggested that the impact on the overall survival may differ depending on the criteria for PD. Therefore, for future clinical trials, it may be necessary to analyze the survival according to the criteria for PD.

Several limitations associated with the present study warrant mention. First, this study was retrospective with a small number of patients and was conducted at a single center. Particularly with regard to the developing patterns of new lesions, our lack of detecting any significant differences may have been due to the small number of patients. Large prospective studies are therefore required to confirm our findings. Second, the regimens used during second-line chemotherapy were not unified. The chemotherapy administered after treatment failure of first-line chemotherapy may therefore have affected the survival. Third, we did not make a detailed evaluation on the degree of increase of the growth of measurable target lesions accompanying the occurrence of new lesions, because the aim of this study was to evaluate the impact of the occurrence of new lesions on the survival, although the degree of increase of the growth of measurable target lesions may affect the survival.

In conclusion, regardless of the developing patterns of new lesions, patients who stopped chemotherapy due to new lesions had worse overall survival rates than those who stopped chemotherapy due only to the growth of measurable target lesions. Because the occurrence of new lesions represents severe progressive disease, patients with new lesions may need an intensive chemotherapy. The type of progression may be useful information to account for when considering subsequent treatment strategies after the failure of first-line chemotherapy.

\section{Acknowledgements}

The authors would like to thank Dr Brian Quinn who assisted in editing the language of this manuscript on behalf of Japan Medical Communication (JMC), Ltd., Japan.

\section{Funding}

No funding was received.

\section{Availability of data and materials}

The datasets used and/or analyzed during the current study are available from the corresponding author on reasonable request.

\section{Authors' contributions}

MS and KM designed the study, performed the statistical analysis and assisted in writing the manuscript. HN, TF and SM collected the clinical data and revised the manuscript 
critically. SK, KH and MO also assisted with designing the study and critically reviewed the manuscript. All authors read and approved the final manuscript.

\section{Ethics approval and consent to participate}

The study was approved by the ethics committee of Osaka City University, and all patients provided written informed consent. All procedures performed in studies involving human participants were in accordance with the Declaration of Helsinki.

\section{Patient consent for publication}

Patients provided written informed consent for the publication of their data.

\section{Competing interests}

The authors declare that they have no competing interests.

\section{References}

1. Therasse P, Arbuck SG, Eisenhauer EA, Wanders J, Kaplan RS, Rubinstein L, Verweij J, Van Glabbeke M, van Oosterom AT, Christian MC and Gwyther SG: New guidelines to evaluate the response to treatment in solid tumors. European organization for research and treatment of cancer, national cancer Institute of the United States, National Cancer Institute of Canada. J Natl Cancer Inst 92: 205-216, 2000.

2. Eisenhauer EA, Therasse P, Bogaerts J, Schwartz LH, Sargent D, Ford R, Dancey J, Arbuck S, Gwyther S, Mooney M, et al: New response evaluation criteria in solid tumours: Revised RECIST guideline (version 1.1). Eur J Cancer 45: 228-247, 2009.

3. Suzuki C, Blomqvist L, Sundin A, Jacobsson H, Byström P, Berglund, A Nygren P and Glimelius B: The initial change in tumor size predicts response and survival in patients with metastatic colorectal cancer treated with combination chemotherapy. Ann Oncol 23: 948-954, 2012.

4. Litière S, de Vries EG, Seymour L, Sargent D, Shankar L and Bogaerts J; RECIST Committee: The components of progression as explanatory variables for overall survival in the response evaluation criteria in solid tumours 1.1 database. Eur J Cancer 50: 1847-1853, 2014.

5. Hurwitz H, Fehrenbacher L, Novotny W, Cartwright T, Hainsworth J, Heim W, Berlin J, Baron A, Griffing S, Holmgren E, et al: Bevacizumab plus irinotecan, fluorouracil, and leucovorin for metastatic colorectal cancer. N Engl J Med 350: 2335-2342, 2004.
6. Venook AP, Niedzwiecki D, Lenz HJ, Innocenti F, Fruth B, Meyerhardt JA, Schrag D, Greene C, O'Neil BH, Atkins JN, et al: Effect of first-line chemotherapy combined with cetuximab or bevacizumab on overall survival in patients with KRAS wild-type advanced or metastatic colorectal cancer: A randomized clinical trial. JAMA 317: 2392-2401, 2017.

7. Gill S, Berry S, Biagi J, Butts C, Buyse M, Chen E, Jonker D, Mărginean C, Samson B, Stewart J, et al: Progression-free survival as a primary endpoint in clinical trials of metastatic colorectal cancer. Curr Oncol 18 (Suppl 2): S5-S10, 2011.

8. Loupakis F, Cremolini C, Masi G, Lonardi S, Zagonel V, Salvatore L, Cortesi E, Tomasello G, Ronzoni M, Spadi R, et al: Initial therapy with FOLFOXIRI and bevacizumab for metastatic colorectal cancer. N Engl J Med 371: 1609-1618, 2014.

9. Yamada Y, Takahari D, Matsumoto H, Baba H, Nakamura M, Yoshida K, Yoshida M, Iwamoto S, Shimada K, Komatsu Y, et al: Leucovorin, fluorouracil, and oxaliplatin plus bevacizumab versus S-1 and oxaliplatin plus bevacizumab in patients with metastatic colorectal cancer (SOFT): An open-label, non-inferiority, randomised phase 3 trial. Lancet Oncol 14: 1278-1286, 2013.

10. Yamazaki K, Nagase M, Tamagawa H, Ueda S, Tamura T, Murata K, Eguchi Nakajima T, Baba E, Tsuda M, Moriwaki T, et al: Randomized phase III study of bevacizumab plus FOLFIRI and bevacizumab plus mFOLFOX6 as first-line treatment for patients with metastatic colorectal cancer (WJOG4407G). Ann Oncol 27: 1539-1546, 2016

11. Tang PA, Bentzen SM, Chen EX and Siu LL: Surrogate end points for median overall survival in metastatic colorectal cancer: Literature-based analysis from 39 randomized controlled trials of first-line chemotherapy. J Clin Oncol 25: 4562-4568, 2007.

12. Buyse M, Burzykowski T, Carroll K, Michiels S, Sargent DJ, Miller LL, Elfring GL, Pignon JP and Piedbois P: Progression-free survival is a surrogate for survival in advanced colorectal cancer. J Clin Oncol 25: 5218-5224, 2007.

13. Van Cutsem E, Köhne CH, Hitre E, Zaluski J, Chang Chien CR, Makhson A, D'Haens G, Pintér T, Lim R, Bodoky G, et al: Cetuximab and chemotherapy as initial treatment for metastatic colorectal cancer. N Engl J Med 360: 1408-1417, 2009.

14. Douillard JY, Siena S, Cassidy J, Tabernero J, Burkes R, Barugel M, Humblet Y, Bodoky G, Cunningham D, Jassem J, et al: Randomized, phase III trial of panitumumab with infusional fluorouracil, leucovorin, and oxaliplatin (FOLFOX4) versus FOLFOX4 alone as first-line treatment in patients with previously untreated metastatic colorectal cancer: The PRIME study. J Clin Oncol 28: 4697-4705, 2010.

15. Cunningham D, Lang I, Marcuello E, Lorusso V, Ocvirk J, Shin DB, Jonker D, Osborne S, Andre N, Waterkamp D, et al: Bevacizumab plus capecitabine versus capecitabine alone in elderly patients with previously untreated metastatic colorectal cancer (AVEX): An open-label, randomised phase 3 trial. Lancet Oncol 14: 1077-1085, 2013. 